\title{
Spectral synthesis on discrete Abelian groups
}

\author{
M. Laczkovich* and L. Székelyhidi ${ }^{\dagger}$
}

April 9, 2008

\begin{abstract}
We prove that spectral synthesis holds on a discrete Abelian group $G$ if and only if the torsion free rank of $G$ is finite.
\end{abstract}

\section{Introduction.}

Let $G$ be a locally compact Abelian group and let $C(G)$ denote the linear space of all complex valued continuous functions defined on $G$ equipped with the topology of uniform convergence on compact sets. By a variety on $G$ we mean a translation invariant closed subspace of $C(G)$. A nonzero continuous function $m \in C(G)$ is called an exponential if $m$ is multiplicative; that is, if $m(x+y)=m(x) \cdot m(y)$ holds for every $x, y \in G$. A function is a polynomial if it belongs to the algebra generated by the continuous additive functions. An exponential monomial is the product of a polynomial and an exponential. If a variety is spanned by exponential monomials, then we say that spectral (or harmonic) synthesis holds for this variety. If spectral synthesis holds for every variety on $G$, then we say that spectral (or harmonic) synthesis holds on $G$.

\footnotetext{
${ }^{1}$ Keywords: problem of spectral synthesis, exponential polynomial

${ }^{2}$ MR subject classification: primary 43A45, 43A 70 , secondary $12 \mathrm{~F} 05$

* Research partially supported by the Hungarian National Foundation for Scientific Research, Grant No. T49786.

${ }^{\dagger}$ Research supported by the Hungarian National Foundation for Scientific Research, Grant No. T043080.
} 
It was proved by M. Lefranc in [7] that for every finite $n$, spectral synthesis holds on the group $\mathbb{Z}^{n}$ equipped with the discrete topology. R. J. Elliot claimed in [2] that spectral synthesis holds on every discrete Abelian group. As it turned out, Elliot's proof was defective. In fact, by a recent result of the second author of this note [11], the statement is false. Let $r_{0}(G)$ denote the torsion free rank of $G$; that is, the cardinality of a maximal independent system of elements of infinite order. In [11] it is shown that spectral synthesis fails on every Abelian group $G$ with $r_{0}(G) \geq \omega$ and a problem is formulated: is it true that if spectral synthesis fails to hold on a discrete Abelian group, then its torsion free rank is at least $\omega$ ? In this paper we answer this question in the affirmative, and thus obtain the following result.

Theorem 1. Spectral synthesis holds on a discrete Abelian group $G$ if and only if $r_{0}(G)$ is finite.

This result also verifies the conjecture formulated by the second author in [12]: spectral synthesis holds on a discrete Abelian group if and only if any complex bi-additive function on the group is a bilinear function of complex additive functions.

As an immediate corollary of Theorem 1 we obtain the following.

Theorem 2. If spectral synthesis holds on the discrete Abelian groups $G$ and $H$, then it also holds on $G \times H$.

It would be interesting to find a direct proof of Theorem 2. In fact, such a proof could simplify the proof of Theorem 1 considerably. As we shall see in Section 3, in order to prove Theorem 1 it is enough to check that spectral synthesis holds on the groups $\mathbb{Q}^{n} \times T$, where $n$ is a positive integer and $T$ is a torsion group. In possession of Theorem 2 it would be enough to prove that spectral synthesis holds on $\mathbb{Q}$ and on every torsion group. The case of torsion groups is relatively simple, using the compactness of their character groups in the product topology (see [1], and Lemma 15 below). Also, the case of $\mathbb{Q}$ is much easier than that of $\mathbb{Q}^{n}$ with $n>1$; this is due to the fact that the structure of ideals of $\mathbb{C}[x]$ is much simpler than those of $\mathbb{C}\left[x_{1}, \ldots, x_{n}\right]$ for $n>1$.

It should be noted that the statement of Theorem 2 is false for locally compact Abelian groups. Indeed, by L. Schwartz's celebrated theorem, spectral synthesis holds on $\mathbb{R}$ with the usual topology (See [10], [4] and [5].) On 
the other hand, as D. I. Gurevič showed, spectral synthesis does not hold on $\mathbb{R}^{2}$ (see [3]). Therefore, any proof of Theorem 2 must use some special properties of varieties on discrete groups. In the next section we present two such properties. First, we show that on discrete groups there is a complete duality between the varieties and the ideals of measures, unlike on some locally compact groups. Also, on discrete groups, the restriction of a variety to a subgroup is again a variety, while on a locally compact group this is not necessarily the case. This latter observation (Theorem 3) is crucial in our proof of Theorem 1.

\section{Preliminaries.}

The linear space $C(G)$ endowed with the topology of uniform convergence on compact sets is a locally convex topological vector space. The set $\mathcal{M}_{c}(G)$ of all signed measures on $G$ having compact support is a ring under the operations of addition and convolution. If $G$ is discrete, then every function is continuous on $G$, and thus $C(G)$ consists of all complex valued functions defined on $G$. Also, in a discrete group a set is compact if and only if it is finite, and thus $\mathcal{M}_{c}(G)$ consists of all measures concentrated on finite sets.

We return to general locally compact Abelian groups. According to the Riesz representation theorem, the continuous linear functionals on $C(G)$ are of the form $f \mapsto \int f d \mu(f \in C(G))$, where $\mu \in \mathcal{M}_{c}(G)$.

Let $V$ be a variety on $G$, and put

$$
V^{\perp}=\left\{\mu \in \mathcal{M}_{c}(G): \int_{G} f d \mu=0 \text { for every } f \in V\right\} .
$$

It is easy to check that $V^{\perp}$ is an ideal of the $\operatorname{ring} \mathcal{M}_{c}(G)$.

If $I \subset \mathcal{M}_{c}(G)$ is an ideal then we shall denote by $I^{\perp}$ the set of functions $f \in C(G)$ such that $\int f d \mu=0$ for every $\mu \in I$. Then $I^{\perp}$ is a variety on $G$. Indeed, it is easy to see that $I^{\perp}$ is a closed linear space. In order to prove translation invariance, let $f \in I^{\perp}, y \in G$ and $\mu \in I$ be arbitrary. If $\delta_{y}$ denotes the Dirac measure concentrated at the point $y$, then we have $\delta_{y} * \mu \in I$, as $I$ is an ideal. Thus

$$
\int_{G} f(x+y) d \mu=\int_{G} f d\left(\delta_{y} * \mu\right)=0
$$


which proves that $I^{\perp}$ is translation-invariant.

We shall also need the fact that $V^{\perp \perp}=V$ for every variety $V$. It is clear that $V \subset V^{\perp \perp}$ for every variety $V$. To prove the other inclusion, suppose that $f \in V^{\perp \perp}$; that is, $\int_{G} f d \mu=0$ for every $\mu \in V^{\perp}$, but $f \notin V$. Then the local convexity of the space implies the existence of a continuous linear functional $L$ such that $L f \neq 0$ and $L g=0$ for every $g \in V$. Let $\mu \in \mathcal{M}_{c}(G)$ be such that $\int_{G} g d \mu=L g$ for every $g \in C(G)$. Then $\mu \in V^{\perp}$ and $\int_{G} f d \mu \neq 0$, a contradiction.

It is obvious that $I \subset I^{\perp \perp}$ holds for every ideal $I \subset \mathcal{M}_{c}(G)$. The inclusion can be proper, as the following simple example shows. Consider $G=\mathbb{R}$ with the usual topology, and let $I$ denote the ideal generated by the measures $\mu_{n}=\delta_{0}-\delta_{1 / n}(n=1,2, \ldots)$. If $f \in I^{\perp}$, then $f$ is periodic mod $1 / n$ for every $n$, and thus, by continuity, $f$ must be constant. Therefore $\delta_{0}-\delta_{\alpha} \in I^{\perp \perp}$ for every $\alpha \in \mathbb{R}$. However, $\delta_{0}-\delta_{\alpha} \notin I$ if $\alpha$ is irrational. Indeed, for every positive integer $N$ there is a continuous function $f$ such that $f$ is periodic $\bmod 1 / n$ for every $n \leq N$ but $f$ is not periodic mod $\alpha$. This easily implies that $\delta_{0}-\delta_{\alpha}$ does not belong to the ideal generated by $\mu_{n}(n \leq N)$. Now $\delta_{0}-\delta_{\alpha} \in I$ would imply that $\delta_{0}-\delta_{\alpha}$ belongs to an ideal generated by finitely many of the measures $\mu_{n}$ which is not the case.

Now we prove that if the group $G$ is discrete, then $I^{\perp \perp}=I$ holds for every ideal $I \subset \mathcal{M}_{c}(G)$. We only have to prove $I^{\perp \perp} \subset I$. If $\nu \in I^{\perp \perp}$, then $\int_{G} f d \nu=0$ for every $f \in I^{\perp}$. Suppose $\nu \notin I$. Since $I$ is a linear subspace of $\mathcal{M}_{c}(G)$ and $\nu \notin I$, there is a linear map $L: \mathcal{M}_{c}(G) \rightarrow \mathbb{C}$ such that $L$ vanishes on $I$ and $L(\nu) \neq 0$. Let $f(x)=L\left(\delta_{x}\right)$ for every $x \in G$. Then

$$
L(\mu)=\int_{G} f d \mu
$$

for every $\mu \in \mathcal{M}_{c}(G)$. Indeed, (1) is true for $\mu=\delta_{x}$ for every $x \in G$ by the definition of $f$. If $G$ is discrete, then every $\mu \in \mathcal{M}_{c}(G)$ is a finite linear combination of measures concentrated on singletons, and thus (1) holds by the linearity of both sides. Now, if $\mu \in I$, then $\int_{G} f d \mu=L(\mu)=0$ by the choice of $L$, and thus $f \in I^{\perp}$. On the other hand, $\int_{G} f d \nu=L(\nu) \neq 0$, which contradicts $\nu \in I^{\perp \perp}$.

Let $G$ be a locally compact Abelian group, and let $V$ be a variety on $G$. If $H$ is a closed subgroup of $G$, then we denote by $\left.V\right|_{H}$ the set of functions $\left\{\left.f\right|_{H}: f \in V\right\}$. 
In general $\left.V\right|_{H}$ need not be a variety, as the following example shows. Let $G=\mathbb{R}$ with the usual topology, and let $\alpha$ be a fixed irrational number. We define $V$ as the set of all functions $f \in C(\mathbb{R})$ which are periodic mod $\alpha$. Clearly, $V$ is a variety on $\mathbb{R}$. We show that $\left.V\right|_{\mathbb{Z}}$ is not a variety on $\mathbb{Z}$, as $\left.V\right|_{\mathbb{Z}}$ is not closed. To prove this, first we show that $\left.V\right|_{\mathbb{Z}}$ is everywhere dense in $C(\mathbb{Z})$. Indeed, let $g: \mathbb{Z} \rightarrow \mathbb{C}$ be an arbitrary function and let $U$ be an arbitrary neighbourhood of $g$. Then there are a positive integer $N$ and a positive number $\varepsilon$ such that $U$ contains every function $h: \mathbb{Z} \rightarrow \mathbb{C}$ for which $|h(n)-g(n)|<\varepsilon$ for every $|n| \leq N$. Now it is easy to see that there exists a function $f \in V$ such that $f(n)=g(n)$ for every $|n| \leq N$. Indeed, the set $S=\{n+k \cdot \alpha:|n| \leq N, k \in \mathbb{Z}\}$ is periodic $\bmod \alpha$, and only has a finite number of elements in the interval $[0, \alpha]$. Let $\left(x_{i}\right)_{i \in \mathbb{Z}}$ be a double infinite sequence such that $S=\left\{x_{i}: i \in \mathbb{Z}\right\}$ and $x_{i-1}<x_{i}$ for every $i$. Let $f(n+k \cdot \alpha)=g(n)$ for every $|n| \leq N$ and $k \in \mathbb{Z}$. Then $f$ is defined on $S$ and is periodic $\bmod \alpha$. Extend $f$ to $\mathbb{R}$ as a linear function in each interval $\left[x_{i-1}, x_{i}\right]$. It is easy to see that $f$ is continuous and is periodic mod $\alpha$; that is, $f \in V$. Since $f(n)=g(n)$ for every $|n| \leq N$, it follows that $\left.f\right|_{\mathbb{Z}} \in U$. This implies that $g$ is in the closure of $\left.V\right|_{\mathbb{Z}}$ and, as $g$ was arbitrary, $\left.V\right|_{\mathbb{Z}}$ is everywhere dense in $C(\mathbb{Z})$. On the other hand, $\left.V\right|_{\mathbb{Z}} \neq C(\mathbb{Z})$, since each element of $\left.V\right|_{\mathbb{Z}}$ is bounded. Therefore, $\left.V\right|_{\mathbb{Z}}$ is not closed and, consequently, is not a variety on $\mathbb{Z}$.

In the next theorem we show that this phenomenon cannot occur in discrete Abelian groups. For every $\mu \in \mathcal{M}_{c}(G)$ the support of $\mu$ will be denoted by $\operatorname{supp} \mu$. Clearly, if $\mu$ has finite support then $\operatorname{supp} \mu=\{x: \mu(\{x\}) \neq 0\}$.

Theorem 3. Let $G$ be a discrete Abelian group, $H$ a subgroup of $G$, and let $V$ be a variety on $G$. Then

(i) $\left.V\right|_{H}$ is a variety on $H$.

(ii) For every function $f: H \rightarrow \mathbb{C}$ we have $\left.f \in V\right|_{H}$ if and only if $\int_{H} f d \mu=$ 0 whenever $\mu \in V^{\perp}$ and $\operatorname{supp} \mu \subset H$.

Proof. First we prove (ii). Suppose that $\left.f \in V\right|_{H}, \mu \in V^{\perp}$ and $\operatorname{supp} \mu \subset H$. Let $g \in V$ be such that $\left.g\right|_{H}=f$, and let $\mu=\sum_{i=1}^{n} a_{i} \delta_{x_{i}}$, where $a_{i} \in \mathbb{C}$ and $x_{i} \in H$ for every $i=1, \ldots, n$. Since $\mu \in V^{\perp}$, we have

$$
\int_{H} f d \mu=\sum_{i=1}^{n} a_{i} \cdot f\left(x_{i}\right)=\sum_{i=1}^{n} a_{i} \cdot g\left(x_{i}\right)=\int_{G} g d \mu=0,
$$


which proves the 'only if' part of the statement.

Next we prove the 'if' part of (ii). Suppose that $f: H \rightarrow \mathbb{C}$ satisfies the condition formulated in (ii); we show $\left.f \in V\right|_{H}$. If $\mu \in \mathcal{M}_{c}(G)$ and $\mu=\sum_{i=1}^{n} a_{i} \delta_{x_{i}}$, then we shall denote by $\phi(\mu)$ the sum of those terms $a_{i} \delta_{x_{i}}$ for which $x_{i} \in G \backslash H$. If there is no such term; that is, if supp $\mu \subset H$, then we define $\phi(\mu)=0$. Clearly, $\phi$ is a linear map from $\mathcal{M}_{c}(G)$ into itself. We put

$$
L(\phi(\mu))=-\int_{H} f d(\mu-\phi(\mu))
$$

for every $\mu \in V^{\perp}$. Then $L$ is well-defined; that is, if $\mu, \nu \in V^{\perp}$ and $\phi(\mu)=$ $\phi(\nu)$, then

$$
\int_{H} f d(\mu-\phi(\mu))=\int_{H} f d(\nu-\phi(\nu)) .
$$

Indeed, $\phi(\mu)=\phi(\nu)$ implies $\phi(\mu-\nu)=0$; that is, $\operatorname{supp}(\mu-\nu) \subset H$. Since $\mu-\nu \in V^{\perp}$, it follows from the condition imposed on $f$ that $\int_{H} f d(\mu-\nu)=0$. Thus $\int_{H} f d \mu=\int_{H} f d \nu$ and, as $\phi(\mu)=\phi(\nu)$, (3) holds.

Therefore, (2) defines a linear map $L: \phi\left(V^{\perp}\right) \rightarrow \mathbb{C}$. Let $\tilde{L}$ be a linear extension of $L$ onto $\mathcal{M}_{c}(G)$, and define

$$
g(x)= \begin{cases}f(x), & \text { if } x \in H, \\ \tilde{L}\left(\delta_{x}\right), & \text { if } x \in G \backslash H .\end{cases}
$$

Then $g$ is an extension of $f$. We show that $g \in V$. If $\mu \in \mathcal{M}_{c}(G)$ and $\mu=\sum_{i=1}^{n} a_{i} \delta_{x_{i}}$, then

$$
\begin{aligned}
\int_{G} g d \mu & =\int_{G \backslash H} g d \mu+\int_{H} g d \mu \\
& =\sum_{x_{i} \in G \backslash H} a_{i} \cdot g\left(x_{i}\right)+\sum_{x_{i} \in H} a_{i} \cdot f\left(x_{i}\right) \\
& =\sum_{x_{i} \in G \backslash H} a_{i} \cdot \tilde{L}\left(\delta_{x_{i}}\right)+\int_{H} f d(\mu-\phi(\mu)) \\
& =\tilde{L}(\phi(\mu))+\int_{H} f d(\mu-\phi(\mu)) .
\end{aligned}
$$

If $\mu \in V^{\perp}$, then (2) gives

$$
\int_{G} g d \mu=\tilde{L}(\phi(\mu))+\int_{H} f d(\mu-\phi(\mu))=0 .
$$


Thus $g \in V^{\perp \perp}=V$ and $f=\left.g\right|_{H}$, which completes the proof of (ii).

Now (i) is an immediate consequence of (ii). Indeed, let $I$ denote the set of measures $\mu \in V^{\perp}$ satisfying $\operatorname{supp} \mu \subset H$, and let $J=\left\{\left.\mu\right|_{H}: \mu \in I\right\}$. Since $V^{\perp}$ is an ideal of $\mathcal{M}_{c}(G)$, it is easy to check that $J$ is an ideal of $\mathcal{M}_{c}(H)$. By (ii) we have $\left.V\right|_{H}=J^{\perp}$, and thus $\left.V\right|_{H}$ is a variety on $H$.

We shall use the following notation. If $f: X \rightarrow \mathbb{C}$ and $g: Y \rightarrow \mathbb{C}$, then $f \otimes g$ will denote the function defined by $f(x) \cdot g(y)$ for every $(x, y) \in X \times Y$.

We shall need the following simple fact about varieties on products, when one of the factors is finite.

Lemma 4. Let $V$ be a variety on $G \times T$, where $G$ is a locally compact Abelian group and $T$ is a finite and discrete Abelian group. Let $|T|=k$, and let $\gamma_{1}, \ldots, \gamma_{k}$ be the characters of $T$. Then for every $g \in V$ there are functions $f_{1}, \ldots, f_{k}: G \rightarrow \mathbb{C}$ such that $f_{i} \otimes \gamma_{i} \in V$ for every $i=1, \ldots, k$, and

$$
g(x, t)=\sum_{i=1}^{k} f_{i}(x) \gamma_{i}(t)
$$

for every $(x, t) \in G \times T$.

Proof. It is well-known that the characters $\gamma_{1}, \ldots, \gamma_{k}$ are linearly independent. Since the set $\mathbb{C}^{T}=\{f: T \rightarrow \mathbb{C}\}$ is a linear space of dimension $k$, it follows that every element of $\mathbb{C}^{T}$ is a linear combination of $\gamma_{1}, \ldots, \gamma_{k}$. Then, for every $x \in G$ there are numbers $f_{1}(x), \ldots, f_{k}(x)$ such that (4) holds for every $t \in T$.

If $T=\left\{t_{j}\right\}_{j=1}^{k}$, then, by the linear independence of $\gamma_{1}, \ldots, \gamma_{k}$ we find that the determinant Det $\left\{\gamma_{i}\left(t_{j}\right)\right\}_{i, j=1}^{k}$ is nonzero. Substituting $t+t_{j}$ for $t$ in (4) we obtain a system of linear equations, from which the unknowns $f_{i}(x) \gamma_{i}(t)$ can be expressed as linear combinations of expressions of the form $g\left(x, t+t_{j}\right)$. As the functions $(x, t) \mapsto g\left(x, t+t_{j}\right)$ belong to $V$, this proves that $f_{i} \otimes \gamma_{i} \in V$ for $i=1, \ldots, k$.

We note that the lemma above easily implies that if spectral synthesis holds on a locally compact Abelian group $G$, then the same is true for $G \times T$ for every finite discrete Abelian group $T$. 


\section{Reduction.}

In this section our aim is to show that if spectral synthesis holds on the groups $\mathbb{Q}^{n} \times T$, where $n$ is a positive integer and $T$ is torsion, then it also holds on every group satisfying $r_{0}(G)<\infty$. This is an immediate consequence of the following two lemmas.

Lemma 5. Every Abelian group with finite torsion free rank can be embedded into a group $\mathbb{Q}^{n} \times T$, where $n=r_{0}(G)$ and $T$ is a torsion group.

Proof. Suppose that $G$ is an Abelian group with $n=r_{0}(G)<\infty$. It is well known [9, Theorem 10.23] that $G$ can be embedded as a subgroup in a divisible group $H$. Let $H_{1}$ denote the set of those elements $x \in H$ for which $m x \in G$ for a suitable positive integer $m$. Clearly, $G \subset H_{1}$. It is easy to check that $H_{1}$ is a divisible subgroup of $H$ and $r_{0}\left(H_{1}\right)=n$. Let $T$ denote the torsion subgroup of $H_{1}$. Then $T$ is also divisible, and thus $T$ is a direct summand of $H_{1}$ by $\left[9\right.$, Corollary 10.10]. Let $H_{1}=H_{2} \times T$, then $H_{2}$ is divisible, torsion free and its torsion free rank is $n$. Consequently, $H_{2}$ is a vector space over $\mathbb{Q}$ of dimension $n$. Thus $H_{2}$ is isomorphic to $\mathbb{Q}^{n}$, which completes the proof.

Lemma 6. If spectral synthesis holds on the discrete Abelian group $G$, then the same is true for every subgroup of $G$.

Proof. Let $H$ be a subgroup of $G$, and let $V \subset C(H)$ be a variety on $H$. Let $T_{x}$ denote the operator of translation by $x$. Then $W=\left\{f \in \mathbb{C}^{G}\right.$ : $\left.\left(T_{x} f\right)\right|_{H} \in V$ for every $\left.x \in G\right\}$ is a variety on $G$. We claim that every function $f \in V$ can be extended to $G$ as an element of $W$. Indeed, let $U$ be a subset of $G$ containing exactly one element of each coset of $H$; we may assume that $U \cap H=\{0\}$. If $f \in V$, then the function $g: G \rightarrow \mathbb{C}$ defined by $g(u+x)=f(x)(u \in U, x \in H)$ belongs to $W$.

By assumption, the set of all exponential polynomials contained by $W$ is dense in $W$. Now, it is easy to check that the restrictions of these exponential monomials to $H$ constitute a dense subset of $V$, which proves that spectral synthesis holds on $H$. 


\section{Modules of polynomials.}

Let $\mathcal{D}$ denote the ring of partial differential operators of $n$ variables. Since the partial derivatives of a polynomial are also polynomials, the ring $\mathbb{C}\left[x_{1}, \ldots, x_{n}\right]$ is a $\mathcal{D}$-module. The submodules of $\mathbb{C}\left[x_{1}, \ldots, x_{n}\right]$ are those linear subspaces of $\mathbb{C}\left[x_{1}, \ldots, x_{n}\right]$ which are closed under partial differentiation. These submodules can be described as follows.

Lemma 7. Let $M$ be a linear subspace of $\mathbb{C}\left[x_{1}, \ldots, x_{n}\right]$. Then the following are equivalent.

(i) $M$ is invariant under translations.

(ii) There exists a nonzero complex number s such that $M$ is invariant under translations by vectors of $s \cdot \mathbb{Z}^{n}$.

(iii) $M$ is a submodule of $\mathbb{C}\left[x_{1}, \ldots, x_{n}\right]$.

Proof. (i) $\Longrightarrow$ (ii) is obvious. Suppose (ii), and let $p \in M$ be arbitrary. If the total degree of $p$ is $d$, then Taylor's formula gives

$$
\sum_{i=0}^{d}(k s)^{i} \cdot \frac{1}{i !} \frac{\partial^{i}}{\partial x_{1}^{i}} p\left(x_{1}, \ldots, x_{n}\right)=p\left(x_{1}+k s, x_{2}, \ldots, x_{n}\right)
$$

for every $k=0,1, \ldots, d$. Now (5) is a system of linear equations with unknowns $\frac{1}{i !} \frac{\partial^{i}}{\partial x_{1}^{i}} p\left(x_{1}, \ldots, x_{n}\right)(i=0, \ldots, d)$. Since the determinant of this system is non-zero, it follows that the partial derivatives $\frac{\partial^{i}}{\partial x_{1}^{i}} p$ are linear combinations of the functions $p\left(x_{1}+k s, x_{2}, \ldots, x_{n}\right)(k=0, \ldots, d)$. By assumption, these functions belong to $M$ for every $k$, and thus $\frac{\partial^{i}}{\partial x_{1}^{i}} p \in M$ for every $i$. By the same argument, $\frac{\partial^{i}}{\partial x_{j}^{i}} p \in M$ for every $j=1, \ldots, n$ and $i \geq 0$; that is, $M$ is a submodule of $\mathbb{C}\left[x_{1}, \ldots, x_{n}\right]$. This proves (ii) $\Longrightarrow($ iii).

The implication (iii) $\Longrightarrow$ (i) is an immediate consequence of Taylor's formula.

The following statement is probably known, but we were unable to find a reference. We are grateful to L. Rónyai for communicating the following proof. 
Lemma 8. The module $\mathbb{C}\left[x_{1}, \ldots, x_{n}\right]$ has the minimal condition. In other words, if $M_{1} \supset M_{2} \supset \ldots$ is a descending sequence of submodules of $\mathbb{C}\left[x_{1}, \ldots, x_{n}\right]$, then there is an $N$ such that $M_{i}=M_{N}$ for every $i \geq N$.

Proof. We order the monomials $x_{1}^{i_{1}} \cdots x_{n}^{i_{n}}$ according to the lexicographic order of the $n$-tuples $\left(i_{1}, \ldots, i_{n}\right)$. By the leading monomial of a nonzero polynomial $p \in \mathbb{C}\left[x_{1}, \ldots, x_{n}\right]$ we mean the largest monomial that appears in $p$ with a nonzero coefficient.

For every submodule $M \subset \mathbb{C}\left[x_{1}, \ldots, x_{n}\right]$ we denote by $S(M)$ the set of leading monomials of the nonzero elements of $M$. It is easy to see, using the fact that $M$ is closed under partial differentiation, that if $m_{1}, m_{2}$ are monomials, $m_{2}$ is a multiple of $m_{1}$ and $m_{2} \in S(M)$, then $m_{1} \in S(M)$.

We show that if $M_{1} \supsetneq M_{2}$, then $S\left(M_{1}\right) \supsetneq S\left(M_{2}\right)$. It is clear that $S\left(M_{1}\right) \supset$ $S\left(M_{2}\right)$. Let $p_{0} \in M_{1} \backslash M_{2}$ have a minimal leading monomial $m_{0}$ among all polynomials $p \in M_{1} \backslash M_{2}$. Then $m_{0} \notin S\left(M_{2}\right)$. Indeed, suppose that $m_{0}$ is a leading monomial of $q \in M_{2}$. Then $p_{0}-c \cdot q \in M_{1} \backslash M_{2}$ for every $c \in \mathbb{C}$, and for a suitable $c$ the leading monomial of $p_{0}-c \cdot q$ is smaller than $m_{0}$, which is impossible. This proves $m_{0} \in S\left(M_{1}\right) \backslash S\left(M_{2}\right)$ and $S\left(M_{1}\right) \supsetneq S\left(M_{2}\right)$.

Now suppose that $M_{1} \supsetneq M_{2} \supsetneq \ldots$ is a strictly decreasing sequence of submodules of $\mathbb{C}\left[x_{1}, \ldots, x_{n}\right]$. Then we have $S\left(M_{1}\right) \supsetneq S\left(M_{2}\right) \supsetneq \ldots$ Let $m_{i} \in S\left(M_{i}\right) \backslash S\left(M_{i+1}\right)$ for every $i \geq 1$. It is not difficult to prove that in every sequence of monomials $\left(m_{i}\right)$ there is an $m_{i}$ which is a multiple of some $m_{j}$ with $j<i$ (see [8, the Theorem on p. 147]). In our case, however, this would imply $m_{j} \in M_{i}$, which contradicts $m_{j} \notin M_{j+1} \supset M_{i}$.

\section{$5 \quad$ Varieties on $\mathbb{Z}^{n}$.}

Let $n$ be a fixed positive integer. In this section we shall write $\mathcal{M}$ for $\mathcal{M}_{c}\left(\mathbb{Z}^{n}\right)$. If $\mu \in \mathcal{M}$ and $\mu=\sum a_{i_{1} \ldots i_{n}} \cdot \delta_{\left(i_{1} \ldots i_{n}\right)}$, then we put

$$
q_{\mu}=\sum a_{i_{1} \ldots i_{n}} \cdot x_{1}^{i_{1}} \cdots x_{n}^{i_{n}} .
$$

The map $\mu \mapsto q_{\mu}$ is a ring isomorphism between $\mathcal{M}$ and the ring $R_{n}$ of functions of the form $x_{1}^{j_{1}} \cdots x_{n}^{j_{n}} \cdot q$, where $j_{1}, \ldots, j_{n} \in \mathbb{Z}$ and $q \in \mathbb{C}\left[x_{1}, \ldots, x_{n}\right]$. If $I \subset \mathcal{M}$, then we put $\widehat{I}=\left\{q_{\mu}: \mu \in I\right\}$. Obviously, if $I$ is an ideal in $\mathcal{M}$, then $\widehat{I}$ is an ideal in $R_{n}$. 
Because of the isomorphism of the rings $\mathcal{M}$ and $R_{n}$ we could actually identify them. We shall not do so; however, if $A$ is an ideal of $R_{n}$, then we shall write

$$
A^{\perp}=\left\{\mu \in \mathcal{M}: q_{\mu} \in A\right\}^{\perp} .
$$

Note that if $A, B$ are ideals of $R_{n}$ and $A \cap \mathbb{C}\left[x_{1}, \ldots, x_{n}\right]=B \cap \mathbb{C}\left[x_{1}, \ldots, x_{n}\right]$, then $A=B$. Indeed, for every $q \in A$ there is a monomial $p=x_{1}^{j_{1}} \cdots x_{n}^{j_{n}}$ such that $p \cdot q$ is a polynomial. Then $p \cdot q \in A \cap \mathbb{C}\left[x_{1}, \ldots, x_{n}\right]=B \cap \mathbb{C}\left[x_{1}, \ldots, x_{n}\right] \subset$ $B$, and hence $q=(p \cdot q) \cdot x_{1}^{-j_{1}} \cdots x_{n}^{-j_{n}} \in B$. Thus $A \subset B$, and the same argument shows $B \subset A$.

Lemma 9. For every positive integer $n$, the family of all varieties on $\mathbb{Z}^{n}$ satisfies the minimal condition. In other words, if $V_{1} \supset V_{2} \supset \ldots$ is a descending sequence of varieties on $\mathbb{Z}^{n}$, then there is an $N$ such that $V_{i}=V_{N}$ for every $i \geq N$.

Proof. If $V_{1} \supset V_{2} \supset \ldots$ is a descending sequence of varieties on $\mathbb{Z}^{n}$, then $V_{1}^{\perp} \subset V_{1}^{\perp} \subset \ldots$ is an ascending sequence of ideals in $\mathcal{M}$. Let $I_{i}=\left(V_{i}^{\perp}\right)^{\wedge} \cap$ $\mathbb{C}\left[x_{1}, \ldots, x_{n}\right]$ for every $i=1,2, \ldots$ Then $I_{1} \subset I_{2} \subset \ldots$ is an ascending sequence of ideals in $\mathbb{C}\left[x_{1}, \ldots, x_{n}\right]$ and thus, as $\mathbb{C}\left[x_{1}, \ldots, x_{n}\right]$ is Noether, there exists an index $N$ such that $I_{i}=I_{N}$ for every $i \geq N$. Then, as we remarked above, we have $\left(V_{i}\right)^{\perp}=\left(V_{N}\right)^{\perp}$ and $V_{i}=\left(V_{N}\right)^{\perp \perp}=\left(V_{N}\right)^{\perp \perp}=V_{N}$ for every $i \geq N$.

If $c=\left(c_{1}, \ldots, c_{n}\right) \in(\mathbb{C} \backslash\{0\})^{n}$, then we shall denote by $m_{c}$ the function

$$
m_{c}\left(x_{1}, \ldots, x_{n}\right)=c_{1}^{x_{1}} \cdots c_{n}^{x_{n}} \quad\left(x_{1}, \ldots, x_{n} \in \mathbb{Z}\right) .
$$

Clearly, $m_{c}$ is an exponential on $\mathbb{Z}^{n}$. A simple calculation shows that

$$
q_{\mu}\left(c_{1}, \ldots, c_{n}\right)=\int_{\mathbb{Z}^{n}} m_{c} d \mu
$$

for every $\mu \in \mathcal{M}$. Let $V$ be a variety on $\mathbb{Z}^{n}$. Then it follows from (7) that $m_{c} \in V$ if and only if $c$ is a root of the ideal $\left(V^{\perp}\right)^{\wedge}$.

In [7] Lefranc gives a condition for $p \cdot m \in V$ for every polynomial $p$ and exponential $m$. The condition is formulated in terms of the partial derivatives of $q_{\mu}\left(\mu \in V^{\perp}\right)$. In order to make the formulas as simple as possible, we shall slightly change the notation used by Lefranc. We denote $x^{[0]}=1$ and 
$x^{[i]}=x \cdot(x-1) \cdots(x-i+1)$ for every $i=1,2, \ldots$ Every $p \in \mathbb{C}\left[x_{1}, \ldots, x_{n}\right]$ can be written uniquely in the form

$$
p=\sum a_{i_{1} \ldots i_{n}} \cdot x_{1}^{\left[i_{1}\right]} \cdots x_{n}^{\left[i_{n}\right]} .
$$

Then we define

$$
D_{p}=\sum a_{i_{1} \ldots i_{n}} \cdot \frac{\partial^{i_{1}+\ldots+i_{n}}}{\partial x_{1}^{i_{1}} \ldots \partial x_{n}^{i_{n}}} \in \mathcal{D} .
$$

Clearly, the map $p \mapsto D_{p}$ is a linear isomorphism from $\mathbb{C}\left[x_{1}, \ldots, x_{n}\right]$ onto $\mathcal{D}$. (Note, however, that $p \mapsto D_{p}$ is a not a ring isomorphism.) Now we prove that

$$
\int_{\mathbb{Z}^{n}} p d \mu=D_{p}\left(q_{\mu}\right)(1, \ldots, 1)
$$

for every $p \in \mathbb{C}\left[x_{1}, \ldots, x_{n}\right]$ and $\mu \in \mathcal{M}$. Since both sides of (8) are linear in both $p$ and $\mu$, it is enough to check the case when $p=x_{1}^{\left[i_{1}\right]} \cdots x_{n}^{\left[i_{n}\right]}$ and $\mu=\delta_{\left(j_{1}, \ldots, j_{n}\right)}$. Then both sides of (8) equals $j_{1}^{\left[i_{1}\right]} \cdots j_{n}^{\left[i_{n}\right]}$, which completes the proof. The next lemma is an immediate corollary of (8).

Lemma 10. Let $V$ be a variety on $\mathbb{Z}^{n}$, and let $p \in \mathbb{C}\left[x_{1}, \ldots, x_{n}\right]$. Then $p \in V$ if and only if $D_{p}\left(q_{\mu}\right)(1, \ldots, 1)=0$ for every $\mu \in V^{\perp}$.

Lemma 11. Let $V$ be a variety on $\mathbb{Z}^{n}$, and let $\left(V^{\perp}\right)^{\wedge}=A \cap B$, where $A$ and $B$ are ideals of $R_{n}$. Suppose that $p \in V$, where $p \in \mathbb{C}\left[x_{1}, \ldots, x_{n}\right]$. If $(1, \ldots, 1)$ is not a root of $B$, then $p \in A^{\perp}$.

Proof. By the previous lemma, it is enough to show that $D_{p}\left(q_{\mu}\right)(1, \ldots, 1)=$ 0 for every measure $\mu$ with $q_{\mu} \in A$. Let $d$ denote the (total) degree of $p$.

Since $(1, \ldots, 1)$ is not a root of $B$, there is a function $t \in B$ such that $t(1, \ldots, 1)=1$. If $s=1-t$, then $1-s^{d+1} \in B$, as

$$
1-s^{d+1}=(1-s)\left(1+s+\ldots+s^{d}\right)=t \cdot\left(1+s+\ldots+s^{d}\right) .
$$

If $q_{\mu} \in A$, then we have

$$
\left(1-s^{d+1}\right) \cdot q_{\mu} \in B \cdot A \subset A \cap B=\left(V^{\perp}\right)^{\wedge} .
$$

Let $\sigma \in \mathcal{M}$ be such that $q_{\sigma}=\left(1-s^{d+1}\right)$. Then $q_{\sigma * \mu}=\left(1-s^{d+1}\right) q_{\mu}$, and thus $\sigma * \mu \in V^{\perp}$ by (9). Therefore, $D_{p}\left(q_{\sigma * \mu}\right)(1, \ldots, 1)=0$ by Lemma 10 . From $q_{\sigma * \mu}=q_{\mu}-s^{d+1} \cdot q_{\mu}$ we obtain

$$
D_{p}\left(q_{\mu}\right)(1, \ldots, 1)=D_{p}\left(s^{d+1} \cdot q_{\mu}\right)(1, \ldots, 1) .
$$


Since $s(1, \ldots, 1)=0$ and the degree of the partial differential operator $D_{p}$ equals $d$, it follows that the right hand side of (10) is zero. Thus $D_{p}\left(q_{\mu}\right)(1, \ldots, 1)=0$, which completes the proof.

Lemma 12. Let $V$ be a variety on $\mathbb{Z}^{n}$, and let $H$ be a subgroup of $\mathbb{Z}^{n}$ of finite index. Then

(i) $M=\left\{p \in \mathbb{C}\left[x_{1}, \ldots, x_{n}\right]:\left.\left.p\right|_{H} \in V\right|_{H}\right\}$ is a submodule of $\mathbb{C}\left[x_{1}, \ldots, x_{n}\right]$.

(ii) If $p \in \mathbb{C}\left[x_{1}, \ldots, x_{n}\right]$ and $\left.\left.p\right|_{H} \in V\right|_{H}$, then $\int_{\mathbb{Z}^{n}} p d(\nu * \mu)=0$ whenever $\nu \in \mathcal{M}, \mu \in V^{\perp}$, and $\operatorname{supp} \mu \subset H$.

Proof. Since $H$ is of finite index, there is a positive integer $N$ such that $N \cdot \mathbb{Z}^{n} \subset H$. It is clear that $M$ is a linear subspace of $\mathbb{C}\left[x_{1}, \ldots, x_{n}\right]$, and that $M$ is invariant under translations by elements of $N \cdot \mathbb{Z}^{n} \subset H$. By Lemma 7 we obtain (i).

In order to prove (ii) we may assume that $\nu=\delta_{\left(j_{1} \ldots j_{n}\right)}$. Then

$$
\int_{\mathbb{Z}^{n}} p d(\nu * \mu)=\int_{\mathbb{Z}^{n}} p\left(x_{1}+j_{1}, \ldots, x_{n}+j_{n}\right) d \mu\left(x_{1}, \ldots, x_{n}\right) .
$$

Since, by (i), the polynomial $p\left(x_{1}+j_{1}, \ldots, x_{n}+j_{n}\right)$ belongs to $\left.V\right|_{H}$, it follows from Theorem 3 that the value of the right hand side of (11) is zero.

For every ideal $A \subset R_{n}$ and $\alpha \in \mathbb{C} \backslash\{0\}$ we define

$$
A(\alpha)=\left\{p \in R_{n}: p\left(\alpha x_{1}, x_{2}, \ldots, x_{n}\right) \in A\right\} ;
$$

it is an ideal of $R_{n}$. If $I$ is an ideal of $\mathcal{M}$, then we shall write $I(\alpha)=\{\mu$ : $\left.q_{\mu} \in \widehat{I}(\alpha)\right\}$; then $I(\alpha)$ is also an ideal of $\mathcal{M}$.

Lemma 13. Let $H=\left\{\left(x_{1}, \ldots, x_{n}\right) \in \mathbb{Z}^{n}: N \mid x_{1}\right\}$, where $N>1$. Let $V$ be a variety on $\mathbb{Z}^{n}$, and let $p \in C\left[x_{1}, \ldots, x_{n}\right]$. Then $\left.\left.p\right|_{H} \in V\right|_{H}$ if and only if

$$
p \in\left(I \cap I(\varepsilon) \cap \ldots \cap I\left(\varepsilon^{N-1}\right)\right)^{\perp},
$$

where $I=V^{\perp}$ and $\varepsilon=e^{2 \pi i / N}$.

Proof. We put $I \cap I(\varepsilon) \cap \ldots \cap I\left(\varepsilon^{N-1}\right)=J$. Suppose $p \in J^{\perp}$; we prove $\left.\left.p\right|_{H} \in V\right|_{H}$. By Theorem 3 it is enough to show that $\int_{H} p d \mu=0$, whenever 
$\mu \in I$ and $\operatorname{supp} \mu \subset H$. For such a $\mu$ the exponent of $x_{1}$ in each term of $q_{\mu}$ is divisible by $N$. Therefore, $q_{\mu}\left(\varepsilon^{j} x_{1}, x_{2}, \ldots, x_{n}\right)=q_{\mu}\left(x_{1}, \ldots, x_{n}\right)$ and thus $\mu \in I\left(\varepsilon^{j}\right)$ for every $j$. Then $\mu \in J$ and hence, by $p \in J^{\perp}$, we obtain $\int_{H} p d \mu=\int_{\mathbb{Z}^{n}} p d \mu=0$. This proves the 'if' direction of the statement.

In order to prove the 'only if' direction, suppose $\left.\left.p\right|_{H} \in V\right|_{H}$, and let $\mu \in J$ be arbitrary. We have to show

$$
\int_{\mathbb{Z}^{n}} p d \mu=0
$$

Let $q=q_{\mu}$, then $q \in \widehat{I}\left(\varepsilon^{j}\right)$ for every $j=0, \ldots, N-1$. We denote by $q_{\ell}$ the sum of those terms of $q$ in which the exponent of $x_{1}$ is congruent to $\ell \bmod N$. Then $q=q_{0}+\ldots+q_{N-1}$. The condition $q \in \widehat{I}\left(\varepsilon^{j}\right)$ gives $q\left(\varepsilon^{j} x_{1}, \ldots, x_{n}\right) \in \widehat{I}$; that is,

$$
q_{0}+\varepsilon^{j} q_{1}+\varepsilon^{2 j} q_{2}+\ldots+\varepsilon^{(N-1) j} q_{N-1}=t_{j} \in \widehat{I}
$$

for every $j=0, \ldots, N-1$. The system of equations (14) has a nonzero determinant, since the numbers $1, \varepsilon, \ldots, \varepsilon^{N-1}$ are different. Thus each $q_{\ell}$ is a linear combination of the functions $t_{j}$, hence $q_{\ell} \in \widehat{I}$ for every $\ell=0, \ldots, N-1$. Let $\mu_{\ell} \in I$ be such that $q_{\mu_{\ell}}=q_{\ell}$. Then $\mu=\mu_{0}+\ldots+\mu_{N-1}$ and thus, in order to prove (13), it is enough to show that $\int_{\mathbb{Z}^{n}} p d \mu_{\ell}=0$ for every $\ell$. Since $q_{\ell} \in \widehat{I}$, we have $x_{1}^{-\ell} \cdot q_{\ell} \in \widehat{I}$, and the exponent of $x_{1}$ in each term of $x_{1}^{-\ell} \cdot q_{\ell}$ is divisible by $N$. If $\delta=\delta_{(-\ell, 0, \ldots, 0)}$, then $\delta * \mu_{\ell} \in I$, and $\operatorname{supp}\left(\delta * \mu_{\ell}\right) \subset H$. Since $\left.p \in V\right|_{H}$, we find, by (ii) of Lemma 12, that

$$
\int_{\mathbb{Z}^{n}} p d \mu_{\ell}=\int_{\mathbb{Z}^{n}} p d\left(\delta_{(\ell, 0, \ldots, 0)} *\left(\delta * \mu_{\ell}\right)\right)=0,
$$

which completes the proof.

Lemma 14. Let $N>1$ and $1 \leq i \leq n$ be integers, and let

$$
H=\left\{\left(x_{1}, \ldots, x_{n}\right) \in \mathbb{Z}^{n}: N\left|x_{1}, \ldots, N\right| x_{i}\right\} .
$$

Let $V$ be a variety on $\mathbb{Z}^{n}$, and let $\left.p \cdot m \in V\right|_{H}$, where $p \in C\left[x_{1}, \ldots, x_{n}\right]$ and $m: H \rightarrow \mathbb{C}$ is an exponential. Then for every finite set $F \subset H$ and for every $\eta>0$ there are polynomials $p_{1}, \ldots, p_{k}$ and exponentials $m_{1}, \ldots, m_{k}$ defined on $\mathbb{Z}^{n}$ such that $\left.m_{j}\right|_{H}=m$ and $p_{j} \cdot m_{j} \in V$ for every $j=1, \ldots, k$, and

$$
\left|p(x)-\sum_{j=1}^{k} p_{j}(x)\right|<\eta
$$

for every $x \in F$. 
Proof. We shall prove the statement by induction on $i$.

I. Let $i=1$; then $H=\left\{\left(x_{1}, \ldots, x_{n}\right) \in \mathbb{Z}^{n}: N \mid x_{1}\right\}$. First we shall assume that $m \equiv 1$ on $H$. Then $\left.\left.p\right|_{H} \in V\right|_{H}$. In $\mathbb{C}\left[x_{1}, \ldots, x_{n}\right]$ every ideal is the intersection of primary ideals, and thus

$$
\left(V^{\perp}\right)^{\wedge} \cap \mathbb{C}\left[x_{1}, \ldots, x_{n}\right]=Q_{1} \cap \ldots \cap Q_{s},
$$

where $Q_{1}, \ldots, Q_{s}$ are primary ideals. We shall prove the lemma by induction on $s$. If $s=0$, then $\left(V^{\perp}\right)^{\wedge}=R_{n}, V^{\perp}=\mathcal{M}, V=\{0\}, p=0$, and thus we can choose $m_{1} \equiv 1$ and $p_{1}=0$.

Suppose that $s>0$, and the statement is true for $s-1$. Let $\varepsilon=e^{2 \pi i / N}$. First we assume that each ideal $Q_{j}$ has a root $c_{j}=\left(\varepsilon^{t_{j}}, 1, \ldots, 1\right)$, where $t_{j}$ is an integer. It is easy to check that $c_{j}$ is also a root of $\left(V^{\perp}\right)^{\wedge}$, and thus the exponential $m_{c_{j}}$ belongs to $V$ for every $j=1, \ldots, s$. Moreover, as Lefranc proved in [7], the set of functions

$$
\left\{\sum_{j=1}^{s} p_{j} \cdot m_{c_{j}}: p_{j} \in \mathbb{C}\left[x_{1}, \ldots, x_{n}\right], p_{j} \cdot m_{c_{j}} \in V(j=1, \ldots, s)\right\}
$$

is everywhere dense in $V$. Since $\left.m_{c_{j}}\right|_{H}=1$ for every $j$, the statement of the lemma is true.

Next suppose that some of the ideals $Q_{j}$ does not have roots of the form $\left(\varepsilon^{t_{j}}, 1, \ldots, 1\right)$. We may assume that $Q_{1}$ is such an ideal.

We put $V^{\perp}=I$ and $J=I \cap I(\varepsilon) \cap \ldots \cap I\left(\varepsilon^{N-1}\right)$. Then, by Lemma 13, we have $p \in J^{\perp}$.

If $A$ is an ideal of $\mathbb{C}\left[x_{1}, \ldots, x_{n}\right]$, then we shall denote by $A^{*}$ the ideal of $R_{n}$ generated by $A$. Obviously,

$$
A^{*}=\left\{x_{1}^{j_{1}} \cdots x_{n}^{j_{n}} \cdot q: j_{1}, \ldots, j_{n} \in \mathbb{Z}, q \in A\right\} .
$$

By (16) we have $\widehat{I}=\left(V^{\perp}\right)^{\wedge}=Q_{1}^{*} \cap \ldots \cap Q_{s}^{*}$. Let $K \subset \mathcal{M}$ be the ideal satisfying $\widehat{K}=Q_{2}^{*} \cap \ldots \cap Q_{s}^{*}$. Then

$$
\widehat{J}=\left[Q_{1}^{*} \cap Q_{1}^{*}(\varepsilon) \cap \ldots \cap Q_{1}^{*}\left(\varepsilon^{N-1}\right)\right] \cap\left[\widehat{K} \cap \widehat{K}(\varepsilon) \cap \ldots \cap \widehat{K}\left(\varepsilon^{N-1}\right)\right] .
$$

Since $Q_{1}^{*}$ does not have roots of the form $\left(\varepsilon^{j}, 1, \ldots, 1\right)$, it follows that $(1, \ldots, 1)$ is not a root of any of the ideals $Q_{1}^{*}\left(\varepsilon^{j}\right)(j=0, \ldots, N-1)$. Let $r_{j} \in Q_{1}^{*}\left(\varepsilon^{j}\right)$ 
be such that $r_{j}(1, \ldots, 1) \neq 0$. Then $r=r_{0} \cdots r_{N-1} \in Q_{1}^{*} \cap Q_{1}^{*}(\varepsilon) \cap \ldots \cap$ $Q_{1}^{*}\left(\varepsilon^{N-1}\right)$ and $r(1, \ldots, 1) \neq 0$. Therefore, $(1, \ldots, 1)$ is not a root of the ideal $Q_{1}^{*} \cap Q_{1}^{*}(\varepsilon) \cap \ldots \cap Q_{1}^{*}\left(\varepsilon^{N-1}\right)$.

As we saw above, $p \in J^{\perp}$. Then (17) and Lemma 11 imply

$$
p \in\left[K \cap K(\varepsilon) \cap \ldots \cap K\left(\varepsilon^{N-1}\right)\right]^{\perp} .
$$

Therefore, by Lemma 13, $\left.\left.p\right|_{H} \in W\right|_{H}$, where $W=K^{\perp}$. Since $I \subset K$, we obtain $W=K^{\perp} \subset I^{\perp}=V$. Also,

$$
\widehat{K} \cap \mathbb{C}\left[x_{1}, \ldots, x_{n}\right]=\left(Q_{2}^{*} \cap \ldots \cap Q_{s}^{*}\right) \cap \mathbb{C}\left[x_{1}, \ldots, x_{n}\right]=R_{2} \cap \ldots \cap R_{s},
$$

where $R_{i}=Q_{i}^{*} \cap \mathbb{C}\left[x_{1}, \ldots, x_{n}\right](2 \leq i \leq s)$. It is easy to check that $R_{2}, \ldots, R_{s}$ are primary ideals. Then, by the induction hypothesis, there are polynomials $p_{1}, \ldots, p_{k}$ and exponentials $m_{1}, \ldots, m_{k}$ such that $p_{j} \cdot m_{j} \in W \subset V$ and $\left.m_{j}\right|_{H} \equiv 1$ for every $j=1, \ldots, k$, and (15) holds for every $x \in F$. This proves the case when $m \equiv 1$.

Now we consider the case when $m$ is an arbitrary exponential on $H$. Let $c_{1} \in \mathbb{C}$ be such that $c_{1}^{N}=m(N, 0, \ldots, 0)$, and let $c_{j}=m(0, \ldots, 0, \underset{j}{1}, 0, \ldots, 0)$ for every $j=2, \ldots, n$. It is easy to see that the exponential $m_{c}$ defined by (6) is an extension of $m$ onto $\mathbb{Z}^{n}$. Let $V_{1}=\left\{f / m_{c}: f \in V\right\}$. Then $V_{1}$ is a variety on $\mathbb{Z}^{n}$, and $\left.\left.p\right|_{H} \in V_{1}\right|_{H}$. Let the finite set $F \subset H$ and $\eta>0$ be given. We put $\eta^{\prime}=\eta / \max _{x \in F}\left|m_{c}(x)\right|$. As we proved above, there are polynomials $p_{1}, \ldots, p_{k}$ and exponentials $m_{1}, \ldots, m_{k}$ such that $p_{j} \cdot m_{j} \in V_{1}$ and $\left.m_{j}\right|_{H} \equiv 1$ for every $j$, and

$$
\left|p(x)-\sum_{j=1}^{k} p_{j}(x)\right|<\eta^{\prime}
$$

for every $x \in F$. Since $p_{j} \cdot\left(m_{j} m_{c}\right) \in V$ by $p_{j} \cdot m_{j} \in V_{1}$ for every $j$, the proof of the case $i=1$ is complete.

II. Now let $1 \leq i<n$, and suppose that the statement of the lemma is true for $i$. We prove the statement for $i+1$. Let

$$
A=\left\{\left(x_{1}, \ldots, x_{n}\right) \in \mathbb{Z}^{n}: N\left|x_{1}, \ldots, N\right| x_{i+1}\right\},
$$

and let $p, m, F, \eta$ be as in the lemma, with $A$ in place of $H$. Let

$$
B=\left\{\left(x_{1}, \ldots, x_{n}\right) \in \mathbb{Z}^{n}: N\left|x_{1}, \ldots, N\right| x_{i}\right\} .
$$


Then $A$ is a subgroup of $B$, and there is an isomorphism $\phi$ from $\mathbb{Z}^{n}$ onto $B$ such that

$$
\phi^{-1}(A)=\left\{\left(x_{1}, \ldots, x_{n}\right) \in \mathbb{Z}^{n}: N \mid x_{1}\right\} .
$$

(We define $\phi\left(x_{1}, \ldots, x_{n}\right)=\left(N x_{2}, \ldots, N x_{i+1}, x_{1}, x_{i+2}, \ldots, x_{n}\right)$ for every $x_{1}, \ldots, x_{n} \in \mathbb{Z}$.) Therefore, the statement of the lemma remains true if we replace $\mathbb{Z}^{n}$ by $B$ and $H$ by $A$. Since $\left.V\right|_{B}$ is a variety on $B$ and $\left.p m \in V\right|_{A}$, we obtain the polynomials $p_{1}, \ldots, p_{k}$ and the exponentials $m_{1}, \ldots, m_{k}$ defined on $B$ such that $\left.m_{j}\right|_{A}=m$ and $\left.p_{j} \cdot m_{j} \in V\right|_{B}$ for every $j=1, \ldots, k$, and

$$
\left|p(x)-\sum_{j=1}^{k} p_{j}(x)\right|<\eta / 2
$$

for every $x \in F$. By the induction hypothesis, the statement of the lemma is true if we replace $H$ by $B$. Thus, for every $j=1, \ldots, k$ we obtain the polynomials $p_{j, \ell}$ and the exponentials $m_{j, \ell}$ defined on $\mathbb{Z}^{n}$ such that $p_{j, \ell} \cdot m_{j, \ell} \in$ $V$ and $\left.m_{j, \ell}\right|_{A}=m_{j}$ for every $\ell=1, \ldots, s_{j}$, and

$$
\left|p_{j}(x)-\sum_{\ell=1}^{s_{j}} p_{j, \ell}(x)\right|<\eta /(2 k)
$$

for every $x \in F$. It is clear that the polynomials $p_{j, \ell}$ and the exponentials $m_{j, \ell}\left(j=1, \ldots, k, \ell=1, \ldots, s_{j}\right)$ satisfy the requirements.

\section{Varieties on $\mathbb{Z}^{n} \times T$, where $T$ is torsion.}

Lemma 15. Spectral synthesis holds on $\mathbb{Z}^{n} \times T$ for every positive integer $n$ and for every discrete torsion group $T$.

Proof. Let $V$ be a variety on $\mathbb{Z}^{n} \times T$, and let $g \in V$ be arbitrary. We have to show that for every finite set $F \subset \mathbb{Z}^{n} \times T$ and for every $\varepsilon>0$ there is an exponential polynomial $\phi \in V$ such that $|\phi(y)-g(y)|<\varepsilon$ for every $y \in F$. Let $|F|=k$, and let $F_{1} \subset \mathbb{Z}^{n}$ and $F_{2} \subset T$ be finite sets such that $F \subset F_{1} \times F_{2}$.

We shall denote by $\mathcal{H}$ the family of all finite subgroups $H \subset T$ satisfying $F_{2} \subset H$. Let $H \in \mathcal{H}$, and let $\gamma_{1}, \ldots, \gamma_{s}$ be the characters of $H$. By Lemma 4 , 
there are functions $f_{1}, \ldots, f_{s}: \mathbb{Z}^{n} \rightarrow \mathbb{C}$ such that $\left.f_{i} \otimes \gamma_{i} \in V\right|_{\mathbb{Z}^{n} \times H}$ for every $i=1, \ldots, s$, and

$$
g(x, t)=\sum_{i=1}^{s} f_{i}(x) \gamma_{i}(t)
$$

for every $(x, t) \in F$. Now, the set of functions $\mathbb{C}^{F}=\{f: F \rightarrow \mathbb{C}\}$ is a linear space of dimension $k$. The functions $\phi_{i}=\left.\left(f_{i} \otimes \gamma_{i}\right)\right|_{F}(i=1, \ldots, s)$ generate a linear subspace $L$ of $\mathbb{C}^{F}$. Thus the dimension of $L$ is at most $k$, and it is generated by at most $k$ of the functions $\phi_{i}$. We may assume that $L$ is generated by the functions $\phi_{1}, \ldots, \phi_{k}$. Since, by (18), $\left.g\right|_{F} \in L$, we have $g(y)=\sum_{i=1}^{k} c_{i} \phi_{i}(y)$ for every $y \in F$ with suitable constants $c_{i}$. Now we have $c_{i} \phi_{i}=\left.\left(c_{i} f_{i}\right) \otimes \gamma_{i} \in V\right|_{\mathbb{Z}^{n} \times H}$ for every $i=1, \ldots, k$. We have proved the following statement: for every $H \in \mathcal{H}$ there are characters $\gamma_{1}, \ldots, \gamma_{k} \in \widehat{H}$ and there are functions $f_{1}, \ldots, f_{k}: \mathbb{Z}^{n} \rightarrow \mathbb{C}$ such that $\left.f_{i} \otimes \gamma_{i} \in V\right|_{\mathbb{Z}^{n} \times H}$ for every $i=1, \ldots, k$, and

$$
g(x, t)=\sum_{i=1}^{k} f_{i}(x) \gamma_{i}(t)
$$

for every $(x, t) \in F$.

If $H \in \mathcal{H}$ and $\gamma \in \widehat{H}$, then we shall denote by $V(\gamma)$ the set of functions $f: \mathbb{Z}^{n} \rightarrow \mathbb{C}$ such that $\left.f \otimes \gamma \in V\right|_{\mathbb{Z}^{n} \times H}$. It is easy to check that $V(\gamma)$ is a variety on $\mathbb{Z}^{n}$. Note that if $H \subset H^{\prime}, \gamma \in \widehat{H}, \gamma^{\prime} \in \widehat{H^{\prime}}$ and $\gamma^{\prime}$ is an extension of $\gamma$, then $V(\gamma) \supset V\left(\gamma^{\prime}\right)$. We shall refer to this property by saying that the variety $V(\gamma)$ is a decreasing function of $\gamma$.

For every $H \in \mathcal{H}$ let $\Gamma_{H}$ denote the set of $k$-tuples $\left(\gamma_{1}, \ldots, \gamma_{k}\right) \in(\widehat{H})^{k}$ such that, for suitable functions $f_{i} \in V\left(\gamma_{i}\right)(i=1, \ldots, k),(19)$ holds for every $(x, t) \in F$. As we proved above, $\Gamma_{H} \neq \emptyset$ for every $H \in \mathcal{H}$.

Our next aim is to prove that there are characters $\chi_{1}, \ldots, \chi_{k} \in \widehat{T}$ with the following property: for every $H \in \mathcal{H}$, we have $\left(\left.\chi_{1}\right|_{H}, \ldots,\left.\chi_{k}\right|_{H}\right) \in \Gamma_{H}$. This will be shown by a compactness argument.

If $\chi \in \widehat{T}$ and $t \in T$ is an element of order $d(t)$, then $\chi(t)$ is a root of unity of order $d(t)$. Consider the product $P=\prod_{t \in T} X_{t}$, where $X_{t}$ is the set of roots of unity of order $d(t)$ equipped with the discrete topology for every $t \in T$. Then $P$ is a compact topological space, and $\widehat{T} \subset P$. The product space $P^{k}$ is also compact. 
For every $H \in \mathcal{H}$, the set $\Gamma_{H}$ is finite. Therefore, the set $C_{H}$ of $k$-tuples $\left(p_{1}, \ldots, p_{k}\right) \in P^{k}$ such that $\left(\left.p_{1}\right|_{H}, \ldots,\left.p_{k}\right|_{H}\right) \in \Gamma_{H}$ is a closed, hence compact subset of $P^{k}$. We show that if $H_{1}, \ldots, H_{m} \in \mathcal{H}$, then $C_{H_{1}} \cap \ldots \cap C_{H_{m}} \neq \emptyset$. Indeed, if $H$ denotes the group generated by $H_{1} \cup \ldots \cup H_{m}$, then $H$ is finite (since $T$ is torsion), and thus $H \in \mathcal{H}$. Let $\left(\gamma_{1}, \ldots, \gamma_{k}\right) \in \Gamma_{H}$, and let $p_{i} \in P$ be an arbitrary extension of $\gamma_{i}$ to $T(i=1, \ldots, k)$. It is clear that $\left(p_{1}, \ldots, p_{k}\right)$ belongs to $C_{H_{j}}$ for every $j=1, \ldots, m$.

Therefore, by the compactness of $P^{k}$, we have $\bigcap_{H \in \mathcal{H}} C_{H} \neq \emptyset$. Let $\left(\chi_{1}, \ldots, \chi_{k}\right)$ be a common element of each $C_{H}(H \in \mathcal{H})$. Then $\chi_{1}, \ldots, \chi_{k}$ are characters of $T$. Indeed, let $t_{1}, t_{1} \in T$ be arbitrary, and let $H \in \mathcal{H}$ be a finite subgroup containing $t_{1}$ and $t_{2}$. Since $\left.\chi_{i}\right|_{H}$ is a character of $H$, we have $\chi_{i}\left(t_{1}+t_{2}\right)=\chi_{i}\left(t_{1}\right) \cdot \chi_{i}\left(t_{2}\right)$, proving that $\chi_{i}$ is multiplicative. We also have $\chi_{i}(t) \in X_{t}$ for every $t \in T$, and thus $\chi_{i}$ does not vanish. Therefore, $\chi_{i}$ is a character for every $i=1, \ldots, k$.

For every fixed $i=1, \ldots, k$, consider the family of varieties $V\left(\left.\chi_{i}\right|_{H}\right)(H \in$ $\mathcal{H})$. Since the varieties on $\mathbb{Z}^{n}$ satisfy the minimal condition, there is a finite group $B_{i} \in \mathcal{H}$ such that $V\left(\left.\chi_{i}\right|_{B_{i}}\right)$ is minimal among these varieties. Now, as the variety $V(\gamma)$ is a decreasing function of $\gamma$, it follows that, whenever $H \in \mathcal{H}$ and $B_{i} \subset H$ then $V\left(\left.\chi_{i}\right|_{H}\right)=V\left(\left.\chi_{i}\right|_{B_{i}}\right)$. Let $B$ denote the group generated by $B_{1}, \ldots, B_{k}$; then $B \in \mathcal{H}$. Then, for every $H \in \mathcal{H}$ with $B \subset H$ we have $V\left(\left.\chi_{i}\right|_{H}\right)=V\left(\left.\chi_{i}\right|_{B}\right)$ for every $i=1, \ldots, k$.

We selected $\chi_{1}, \ldots, \chi_{k}$ in such a way that $\left(\left.\chi_{1}\right|_{B}, \ldots,\left.\chi_{k}\right|_{B}\right) \in \Gamma_{B}$ holds; that is, there are functions $f_{i} \in V\left(\left.\chi_{i}\right|_{B}\right)$ such that

$$
g(x, t)=\sum_{i=1}^{k} f_{i}(x) \chi_{i}(t)
$$

for every $(x, t) \in F$. As we proved above, we have $V\left(\left.\chi_{i}\right|_{B}\right)=V\left(\left.\chi_{i}\right|_{H}\right)$ for every finite group $H \supset B$, and thus $\left.f_{i} \otimes\left(\left.\chi_{i}\right|_{H}\right) \in V\right|_{\mathbb{Z}^{n} \times H}$ for every such $H$. In other words, if $H \in \mathcal{H}$ and $B \subset H$, then there is a function $g_{H} \in V$ such that $f_{i}(x) \chi_{i}(t)=g_{H}(x, t)\left((x, t) \in \mathbb{Z}^{n} \times H\right)$. In particular, for every finite subset of $\mathbb{Z}^{n} \times T$, the function $f_{i} \otimes \chi_{i}$ equals the restriction of an element of $V$ to the given finite set. Since $V$ is closed, this means that $f_{i} \otimes \chi_{i} \in V$.

We have proved the existence of functions $f_{i}$ and characters $\chi_{i} \in \widehat{T}$ such that $f_{i} \otimes \chi_{i} \in V$ for every $i=1, \ldots, k$, and (20) holds for every $(x, t) \in F$. Let $W_{i}$ denote the set of functions $f: \mathbb{Z}^{n} \rightarrow \mathbb{C}$ such that $f \otimes \chi_{i} \in V$. Then $W_{i}$ is a variety on $\mathbb{Z}^{n}$ containing $f_{i}$. By Lefranc's theorem [7], spectral synthesis 
holds on $\mathbb{Z}^{n}$, and thus we can find, for every given $\varepsilon>0$, an exponential polynomial $\phi_{i} \in W_{i}$ such that $\left|f_{i}(x)-\phi_{i}(x)\right|<\varepsilon / k$ for every $x \in F_{1}$. It is clear that $\phi_{i} \otimes \chi_{i}$ is an exponential polynomial on $\mathbb{Z}^{n} \times T$ and we also have $\phi_{i} \otimes \chi_{i} \in V$ by $\phi_{i} \in W_{i}$. Then $\phi=\sum_{i=1}^{k}\left(\phi_{i} \otimes \chi_{i}\right)$ is an exponential polynomial belonging to $V$ such that $|\phi(y)-g(y)|<\varepsilon$ for every $y \in F$. This completes the proof.

Remark 16. Lemma 15 actually gives the following. If $V$ is a variety on $\mathbb{Z}^{n} \times T$, then $V$ is spanned by all functions in $V$ having the form $(p m) \otimes \chi$, where $p$ is a polynomial, $m$ is an exponential on $\mathbb{Z}^{n}$ and $\chi$ is a character of $T$.

Lemma 17. Let $n, N$ be positive integers, and let

$$
H=N \cdot \mathbb{Z}^{n}=\left\{\left(x_{1}, \ldots, x_{n}\right) \in \mathbb{Z}^{n}: N \mid x_{i}(i=1, \ldots, n)\right\} .
$$

Let $T$ be a discrete torsion group, and let $V$ be a variety on $\mathbb{Z}^{n} \times T$. Suppose that $\left.(p \cdot m) \otimes \chi \in V\right|_{H \times T}$, where $p \in C\left[x_{1}, \ldots, x_{n}\right], m: H \rightarrow \mathbb{C}$ is an exponential, and $\chi \in \widehat{T}$. Then for every finite set $F \subset H \times T$ with $|F|=k$ and for every $\eta>0$ there are polynomials $p_{1}, \ldots, p_{k}$ and exponentials $m_{1}, \ldots, m_{k}$ defined on $\mathbb{Z}^{n}$ such that $\left.m_{j}\right|_{H}=m$ and $\left(p_{j} \cdot m_{j}\right) \otimes \chi \in V$ for every $j=1, \ldots, k$, and

$$
\left|p(x)-\sum_{j=1}^{k} p_{j}(x)\right|<\eta
$$

for every $(x, t) \in F$.

Proof. First we assume that $T$ is finite. Let $F_{1} \subset H$ be a finite set such that $F \subset F_{1} \times T$ and $\left|F_{1}\right| \leq k$. Let $\chi_{1}, \ldots, \chi_{s}$ be the characters of $T$ listed in such a way that $\chi=\chi_{1}$. Since $\left.(p \cdot m) \otimes \chi \in V\right|_{H \times T}$, there exists a $g \in V$ such that $(p \cdot m) \otimes \chi=\left.g\right|_{H \times T}$. By Lemma 4 , there are functions $f_{1}, \ldots, f_{s}: \mathbb{Z}^{n} \rightarrow \mathbb{C}$ such that $f_{i} \otimes \chi_{i} \in V$ for every $i=1, \ldots, s$, and

$$
g(x, t)=\sum_{i=1}^{s} f_{i}(x) \chi_{i}(t)
$$

for every $(x, t) \in \mathbb{Z}^{n} \times T$. If $x \in H$, then (22) gives

$$
p(x) m(x) \chi(t)=\sum_{i=1}^{s} f_{i}(x) \chi_{i}(t)
$$


for every $t \in T$. Since the characters $\chi_{i}$ are linearly independent and $\chi=\chi_{1}$, we find that $p(x) m(x) \chi(t)=f_{1}(x) \chi(t)$ for every $x \in H$. Therefore, we have $p m=\left.f_{1}\right|_{H}$.

Let $V(\chi)$ denote the set of functions $f: \mathbb{Z}^{n} \rightarrow \mathbb{C}$ such that $f \otimes \chi \in V$. Then $V(\chi)$ is a variety on $\mathbb{Z}^{n}$. Since $f_{1} \in V(\chi)$, we have $\left.p m \in V(\chi)\right|_{H}$. Thus, we may apply Lemma 14 , and obtain the polynomials $p_{1}, \ldots, p_{r}$ and the exponentials $m_{1}, \ldots, m_{r}$ defined on $\mathbb{Z}^{n}$ such that $p_{i} m_{i} \in V(\chi)$ and $\left.m_{i}\right|_{H}=m$ for every $i=1, \ldots, r$, and

$$
\left|p(x)-\sum_{i=1}^{r} p_{i}(x)\right|<\eta
$$

for every $x \in F_{1}$. By $p_{i} m_{i} \in V(\chi)$ we have $\left(p_{i} m_{i}\right) \otimes \chi \in V$ for every $i$. Since $F \subset F_{1} \times T$, this proves the statement of the lemma, apart from the fact that $r$ can be larger than $k=|F|$. In order to reduce the number $r$ we may apply the argument used in the previous proof. The set of functions $\mathbb{C}^{F_{1}}=\left\{f: F_{1} \rightarrow \mathbb{C}\right\}$ is a linear space of dimension $\left|F_{1}\right| \leq k$. The functions $\psi_{i}=\left.\left(p_{i} m_{i}\right)\right|_{F_{1}}(i=1, \ldots, r)$ generate a linear subspace $L$ of $\mathbb{C}^{F_{1}}$. Thus the dimension of $L$ is at most $k$, and it is generated by at most $k$ of the functions $\psi_{i}$. We may assume that $L$ is generated by the functions $\psi_{1}, \ldots, \psi_{k}$. Since

$$
S=\left.\left(\sum_{i=1}^{r} p_{i} m_{i}\right)\right|_{F_{1}} \in L,
$$

we have $S(x)=\sum_{i=1}^{k} c_{i} \psi_{i}(x)$ for every $x \in F_{1}$ with suitable constants $c_{i}$. Now we have $c_{i} \psi_{i}=\left(c_{i} p_{i}\right) \cdot m_{i} \in V$ for every $i=1, \ldots, k$, which completes the proof in the case when $T$ is finite.

Now let $T$ be an arbitrary torsion group. Let $F_{1} \subset H$ and $F_{2} \subset T$ be finite sets such that $F \subset F_{1} \times F_{2}$. We shall denote by $\mathcal{H}$ the family of all finite subgroups $G \subset T$ satisfying $F_{2} \subset G$. If $G \in \mathcal{H}$ and $\gamma \in \widehat{G}$, then we shall denote by $V(\gamma)$ the set of functions $f: \mathbb{Z}^{n} \rightarrow \mathbb{C}$ such that $\left.f \otimes \gamma \in V\right|_{\mathbb{Z}^{n} \times G}$. It is easy to check that $V(\gamma)$ is a variety on $\mathbb{Z}^{n}$. Note that if $G \subset G^{\prime}, \gamma \in \widehat{G}, \gamma^{\prime} \in \widehat{G^{\prime}}$ and $\gamma^{\prime}$ is an extension of $\gamma$, then $V(\gamma) \supset V\left(\gamma^{\prime}\right)$. We shall refer to this property by saying that the variety $V(\gamma)$ is a decreasing function of $\gamma$.

For every $G \in \mathcal{H}$ we define two sets of $k$-tuples as follows. $D_{G}$ will denote the set of $k$-tuples $\left(p_{1} m_{1}, \ldots, p_{k} m_{k}\right)$, where $p_{i} \in \mathbb{C}\left[x_{1}, \ldots, x_{n}\right], m_{i}$ is an 
exponential defined on $\mathbb{Z}^{n},\left.m_{i}\right|_{H}=m$ and $\left.\left(p_{i} m_{i}\right) \otimes\left(\left.\chi\right|_{G}\right) \in V\right|_{\mathbb{Z}^{n} \times G}$ for every $i=1, \ldots, k$, and $(21)$ holds for every $(x, t) \in F$. Also, we shall denote by $E_{G}$ the set of those $k$-tuples $\left(m_{1}, \ldots, m_{k}\right)$ for which $\left(p_{1} m_{1}, \ldots, p_{k} m_{k}\right) \in D_{G}$ for some polynomials $p_{1}, \ldots, p_{k}$. As we proved above, $D_{G}$ and $E_{G}$ are nonempty for every $G \in \mathcal{H}$. It is clear that if $G, G^{\prime} \in \mathcal{H}$ and $G \subset G^{\prime}$ then $D_{G} \supset D_{G^{\prime}}$ and $E_{G} \supset E_{G^{\prime}}$. Therefore, the intersections $D_{G_{1}} \cap \ldots \cap D_{G_{s}}$ and $E_{G_{1}} \cap \ldots \cap E_{G_{s}}$ are nonempty for any $G_{1}, \ldots, G_{s} \in \mathcal{H}$.

We prove that $\bigcap\left\{D_{G}: G \in \mathcal{H}\right\} \neq \emptyset$. Since $H=N \cdot \mathbb{Z}^{n}$, it follows that $m$ only hashas only a finite number of extensions to $\mathbb{Z}^{n}$ as an exponential. Indeed, if $m^{\prime}$ is an exponential on $\mathbb{Z}^{n}$ with $\left.m^{\prime}\right|_{H}=m$ then for every $x \in$ $\mathbb{Z}^{n}$ the equation $m^{\prime}(x)^{N}=m^{\prime}(N x)=m(N x)$ implies that the number of possible values of $m^{\prime}(x)$ is at most $N$. Since $\mathbb{Z}^{n}$ is generated by $n$ elements, it follows that the number of possible exponentials defined on $\mathbb{Z}^{n}$ is at most $N^{n}$. Therefore, the set $\bigcup\left\{E_{G}: G \in \mathcal{H}\right\}$ is finite. Then $\bigcap\left\{E_{G}: G \in \mathcal{H}\right\}=$ $E_{G_{1}} \cap \ldots \cap E_{G_{s}}$ for some $G_{1}, \ldots, G_{s} \in \mathcal{H}$, and thus $\bigcap\left\{E_{G}: G \in \mathcal{H}\right\} \neq \emptyset$.

Let $\left(m_{1}, \ldots, m_{k}\right) \in \bigcap\left\{E_{G}: G \in \mathcal{H}\right\}$ be fixed. For every $G \in \mathcal{H}$ we shall denote by $M_{i}(G)$ the set of polynomials $p \in \mathbb{C}\left[x_{1}, \ldots, x_{n}\right]$ such that $\left.\left(p \cdot m_{i}\right) \otimes\left(\left.\chi\right|_{G}\right) \in V\right|_{\mathbb{Z}^{n} \times G}$. Clearly, $M_{i}(G)$ is a linear subspace of $\mathbb{C}\left[x_{1}, \ldots, x_{n}\right]$. We show that $M_{i}(G)$ is invariant under translations by vectors of $\mathbb{Z}^{n}$. Indeed, let $p \in M_{i}(G)$ and $a \in \mathbb{Z}^{n}$ be arbitrary, and let $f \in V$ be such that

$$
\left.f\right|_{\mathbb{Z}^{n} \times G}=\left(p \cdot m_{i}\right) \otimes\left(\left.\chi\right|_{G}\right) .
$$

Since $V$ is translation invariant, the function $(x, t) \mapsto f(x+a, t)$ belongs to $V$. Thus

$p(x+a) \cdot m_{i}(x) \chi(t)=\frac{1}{m_{i}(a)} \cdot p(x+a) \cdot m_{i}(x+a) \chi(t)=\frac{1}{m_{i}(a)} \cdot f(x+a, t)$

for every $(x, t) \in \mathbb{Z}^{n} \times G$, which proves that the polynomial $x \mapsto p(x+a)$ belongs to $M_{i}(G)$.

Therefore, by Lemma $7, M_{i}(G)$ is a submodule of $\mathbb{C}\left[x_{1}, \ldots, x_{n}\right]$. It is easy to see that $M_{i}(G)$ is a decreasing function of $G$.

For every fixed $i=1, \ldots, k$, consider the family of modules $M_{i}(G)(G \in$ $\mathcal{H})$. Since the submodules of $\mathbb{C}\left[x_{1}, \ldots, x_{n}\right]$ satisfy the minimal condition, there is a finite group $B_{i} \in \mathcal{H}$ such that $M_{i}\left(B_{i}\right)$ is minimal among these modules. Now, as the module $M_{i}(G)$ is a decreasing function of $G$, it follows that, whenever $G \in \mathcal{H}$ and $B_{i} \subset G$ then $M_{i}(G)=M_{i}\left(B_{i}\right)(i=1, \ldots, k)$. 
Let $B$ denote the group generated by $B_{1}, \ldots, B_{k}$; then $B \in \mathcal{H}$, and for every $G \in \mathcal{H}$ with $B \subset G$ we have $M_{i}(G)=M_{i}(B)$ for every $i=1, \ldots, k$.

Since $\left(m_{1}, \ldots, m_{k}\right) \in E_{B}$, there are polynomials $p_{1}, \ldots, p_{k}$ such that $\left(p_{1} m_{1}, \ldots, p_{k} m_{k}\right) \in D_{B}$. Then $\left.\left(p_{i} m_{i}\right) \otimes\left(\left.\chi\right|_{B}\right) \in V\right|_{\mathbb{Z}^{n} \times B}$; that is, $p_{i} \in M_{i}(B)$ for every $i=1, \ldots, k$. Now we prove that

$$
\left(p_{1} m_{1}, \ldots, p_{k} m_{k}\right) \in D_{G}
$$

for every $G \in \mathcal{H}$. Let $G \in \mathcal{H}$ be arbitrary; we may assume that $B \subset G$. Then $M_{i}(B)=M_{i}(G)$, and thus $p_{i} \in M_{i}(G)(i=1, \ldots, k)$, which implies (25). Therefore, $\left.\left(p_{i} m_{i}\right) \otimes\left(\left.\chi\right|_{G}\right) \in V\right|_{\mathbb{Z}^{n} \times G}$ for every $G \in \mathcal{H}$. In other words, if $G \in \mathcal{H}$ and $B \subset G$, then there is a function $g_{G} \in V$ such that $p_{i}(x) m_{i}(x) \chi(t)=$ $g_{G}(x, t)\left((x, t) \in \mathbb{Z}^{n} \times G\right)$. In particular, for every finite subset of $\mathbb{Z}^{n} \times T$, the function $p_{i}(x) m_{i}(x) \chi(t)$ equals the restriction of an element of $V$ to the given finite set. Since $V$ is closed, this means that $\left(p_{i} m_{i}\right) \otimes \chi \in V$. This completes the proof of the lemma.

\section{Spectral synthesis on $\mathbb{Q}^{n} \times T$, where $T$ is torsion.}

Let $T$ be a discrete torsion group. In this section we complete the proof of Theorem 1 by showing that spectral synthesis holds on $\mathbb{Q}^{n} \times T$ for every positive integer $n$. Let

$$
G_{i}=\left\{\left(\frac{k_{1}}{i !}, \ldots, \frac{k_{n}}{i !}\right): k_{1}, \ldots, k_{n} \in \mathbb{Z}\right\}
$$

for every $i=1,2, \ldots$ Then $G_{1}, G_{2}, \ldots$ is an increasing sequence of subgroups of $\mathbb{Q}^{n}$ such that $\bigcup_{i=1}^{\infty} G_{i}=\mathbb{Q}^{n}$. In addition, for every $i<j$ there exists a isomorphism $\phi$ from $G_{j}$ onto $\mathbb{Z}^{n}$ and there is an integer $N>1$ such that

$$
\phi\left(G_{i}\right)=\left\{\left(x_{1}, \ldots, x_{n}\right) \in \mathbb{Z}^{n}: N\left|x_{1}, \ldots, N\right| x_{n}\right\} .
$$

This implies that the statement of Lemma 17 remains valid if we replace $\mathbb{Z}^{n}$ by $G_{j}$ and $H$ by $G_{i}$.

Now let $V$ be a variety on $\mathbb{Q} \times T$, and let $g \in V$ be arbitrary. We have to show that for every finite set $F \subset \mathbb{Q}^{n} \times T$ and for every $\varepsilon>0$ there is a exponential polynomial $\phi \in V$ such that $|\phi(y)-g(y)|<\varepsilon$ for every $y \in F$. 
Let $|F|=k$, and let $i_{0}$ be such that $F \subset G_{i_{0}} \times T$. By Theorem $3,\left.V\right|_{G_{i_{0}} \times T}$ is a variety on $G_{i_{0}}$. Since $G_{i_{0}}$ is isomorphic to $\mathbb{Z}^{n}$, it follows from Lemma 15 that spectral synthesis holds in $\left.V\right|_{G_{i_{0}} \times T}$. In other words, we can find a exponential polynomial $\left.\phi_{0} \in V\right|_{G_{i_{0}} \times T}$ such that $\left|g(y)-\phi_{0}(y)\right|<\varepsilon / 2$ for every $y \in F$. Moreover, by Remark 16, we may assume that $\phi_{0}(x, t)=\sum_{i=1}^{s}\left(p_{i} \cdot m_{i}\right) \otimes \chi_{i}$, where $p_{i} \in \mathbb{C}\left[x_{1}, \ldots, x_{n}\right], m_{i}$ is an exponential defined on $G_{i_{0}}, \chi \in \widehat{T}$, and $\left.\left(p_{i} \cdot m_{i}\right) \otimes \chi_{i} \in V\right|_{G_{i_{0}} \times T}$ for every $i=1, \ldots, s$. Clearly, it is enough to show that for every $i$ there is an exponential polynomial $\phi_{i} \in V$ such that

$$
\left|\left(p_{i} \cdot m_{i}\right)(x) \chi_{i}(t)-\phi_{i}(x, t)\right|<\varepsilon /(2 s)
$$

for every $(x, t) \in F$. Summing up: we have to show that whenever

- $p$ is a polynomial,

- $m$ is an exponential on $G_{i_{0}}$,

- $\chi$ is a character of $T$,

- $\left.(p m) \otimes \chi \in V\right|_{G_{i_{0}} \times T}$,

- $F \subset G_{i_{0}} \times T$ is a finite set, and

- $\varepsilon>0$,

then there is an exponential polynomial $\psi \in V$ such that $\mid(p \cdot m)(x) \chi(t)-$ $\psi(x, t) \mid<\varepsilon$ for every $(x, t) \in F$. In the course of the following proof $p, m, \chi, F$ and $\varepsilon$ will be fixed. Let $k$ denote the cardinality of $F$. We put $\eta=\varepsilon / \max _{(x, t) \in F}|m(x)|$.

Let $i>i_{0}$ be given. As we remarked above, the statement of Lemma 17 remains valid if we replace $\mathbb{Z}^{n}$ by $G_{i}$ and $H$ by $G_{i_{0}}$. Since $\left.V\right|_{G_{i} \times T}$ is a variety and $\left.(p m) \otimes \chi \in V\right|_{G_{i_{0}} \times T}$, we obtain the polynomials $p_{1}, \ldots, p_{k}$ and the exponentials $m_{1}, \ldots, m_{k}$ defined on $G_{i}$ such that $\left.m_{j}\right|_{G_{0}}=m$ and $\left.\left(p_{j} m_{j}\right) \otimes \chi \in V\right|_{G_{i} \times T}$ for every $j=1, \ldots, k$, and

$$
\left|p(x)-\sum_{j=1}^{k} p_{j}(x)\right|<\eta
$$

for every $(x, t) \in F$. Let $\Xi$ denote the set of $k+1$-tuples $\left(i, m_{1}, \ldots, m_{k}\right)$ with the following properties: (i) $i \geq i_{0}$; (ii) $m_{1}, \ldots, m_{k}$ are exponentials defined 
on $G_{i}$ such that $\left.m_{j}\right|_{G_{i_{0}}}=m$ for every $j=1, \ldots, k$; (iii) there are polynomials $p_{1}, \ldots, p_{k}$ such that $\left.\left(p_{j} m_{j}\right) \otimes \chi \in V\right|_{G_{i} \times T}$ for every $j=1, \ldots, k$, and (26) holds for every $x \in F$. As we proved, $\Xi$ contains $k+1$-tuples $\left(i, m_{1}, \ldots, m_{k}\right)$ for every $i \geq i_{0}$.

We define a partial order on $\Xi$ as follows: if $\left(i, m_{1}, \ldots, m_{k}\right)$ and $\left(i^{\prime}, m_{1}^{\prime}, \ldots, m_{k}^{\prime}\right)$ are elements of $\Xi$ then we write

$$
\left(i, m_{1}, \ldots, m_{k}\right)<\left(i^{\prime}, m_{1}^{\prime}, \ldots, m_{k}^{\prime}\right)
$$

if $i<i^{\prime}$ and $\left.m_{j}^{\prime}\right|_{G_{i}}=m_{j}$ for every $j=1, \ldots, k$. It is clear that this partial order makes $\Xi$ a tree. Every level of $\Xi$ is finite. Indeed, if $i$ is fixed then there is an integer $N$ such that $N \cdot G_{i}=G_{i_{0}}$. This implies that $m$ only has a finite number of extensions to $G_{i}$ as an exponential, and thus the number of $k+1$-tuples $\left(i, m_{1}, \ldots, m_{k}\right) \in \Xi$ is finite.

By König's lemma [6, 5.7. Lemma, p. 69], $\Xi$ has an infinite chain. That is, there is a sequence of $k+1$-tuples $\left(i, m_{1}^{i}, \ldots, m_{k}^{k}\right) \in \Xi\left(i \geq i_{0}\right)$ such that

$$
\left.m_{j}^{i+1}\right|_{G_{i}}=m_{j}^{i}
$$

for every $i \geq i_{0}$ and $j=1, \ldots, k$.

For every $i \geq i_{0}$ and $j=1, \ldots, k$ we shall denote by $M_{j}^{i}$ the set of polynomials $p \in \mathbb{C}\left[x_{1}, \ldots, x_{n}\right]$ such that $\left.\left(p \cdot m_{j}^{i}\right) \otimes \chi \in V\right|_{G_{i} \times T}$. It is obvious that $M_{j}^{i}$ is a linear subspace of $\mathbb{C}\left[x_{1}, \ldots, x_{n}\right]$. It is easy to see that $M_{j}^{i}$ is invariant under translations by elements of $G_{i}$. Therefore, by Lemma $7, M_{j}^{i}$ is a submodule of $\mathbb{C}\left[x_{1}, \ldots, x_{n}\right]$ for every $i$ and $j$.

For every $i$ and $j$ we have $M_{j}^{i} \supset M_{j}^{i+1}$. Indeed, if $p \in M_{j}^{i+1}$, then $(p$. $\left.m_{j}^{i+1}\right)\left.\otimes \chi \in V\right|_{G_{i+1} \times T}$. By $(27)$, this implies $\left.\left(p \cdot m_{j}^{i}\right) \otimes \chi \in V\right|_{G_{i} \times T}$; that is, $p \in M_{j}^{i}$. Now it follows from Lemma 8 , that for every $j$ there is an index $i(j)$ such that $M_{j}^{i}=M_{j}^{i(j)}$ for every $i \geq i(j)$. Let $i_{1}=\max \left\{i_{0}, i(1), \ldots, i(k)\right\}$. Then we have $M_{j}^{i}=M_{j}^{i_{1}}$ for every $i \geq i_{1}$.

By the definition of $\Xi$ we can find polynomials $p_{j} \in M_{j}^{i_{1}}(j=1, \ldots, k)$ such that (26) holds for every $x \in F$. If $i>i_{1}$ then $M_{j}^{i}=M_{j}^{i_{1}}$ for every $j$, and hence $\left.\left(p_{j} \cdot m_{j}^{i}\right) \otimes \chi \in V\right|_{G_{i} \times T}$ for every $i>i_{1}$ and $j=1, \ldots, k$.

It follows from $(27)$ that for every $j=1, \ldots, k$ there is a function $m_{j}$ : $\mathbb{Q}^{n} \rightarrow \mathbb{C}$ such that $\left.m_{j}\right|_{G_{i}}=m_{j}^{i}$ for every $i \geq i_{1}$. It is clear that $m_{j}$ is an exponential on $\mathbb{Q}^{n}$. By $\left.\left(p_{j} m_{j}^{i}\right) \otimes \chi \in V\right|_{G_{i} \times T}$ we can find a function $g_{j}^{i} \in V$ 
such that $\left.g_{j}^{i}\right|_{G_{i}}=\left(p_{j} m_{j}^{i}\right) \otimes \chi$. It is easy to see that the sequence $\left(g_{j}^{i}\right)_{i \geq i_{1}}$ converges pointwise to the function $\left(p_{j} m_{j}\right) \otimes \chi$, and thus $\left(p_{j} m_{j}\right) \otimes \chi \in V$.

Now $\psi=\sum\left(p_{j} m_{j}\right) \otimes \chi$ is an exponential polynomial belonging to $V$. Taking into consideration that $m_{j}$ is an extension of $m_{j}^{i_{1}}$ and that $F \subset$ $G_{i_{0}} \times T \subset G_{i_{1}} \times T$, it follows from $(26)$ that $|(p \cdot m)(x) \chi(t)-\psi(x, t)|<\varepsilon$ for every $x \in F$. This completes the proof. 


\section{References}

[1] Á. Bereczky and L. Székelyhidi, Spectral synthesis on torsion groups, J. Math. Anal. and Applications 304 (2005), 607-613.

[2] R. J. Elliot, Two notes on spectral synthesis for discrete Abelian groups, Proc. Cambridge Phil. Soc. 61 (1965), 617-620.

[3] D. I. Gurevič, Counterexamples to a problem of L. Schwartz, Funkcional. Anal. i Priložen 9 (1975), no. 2, 29-35. (English translation: Funkcional Anal. Appl. 9 (1975), no. 2, 116-120.)

[4] J.-P. Kahane, Sur quelques problèmes d'unicité et de prolongement, relatifs aux fonctions approchables par des sommes d'exponentielles, Ann. Inst. Fourier (Grenoble) 5 (1953-54), 39-130.

[5] J.-P. Kahane: Lectures on Mean Periodic Functions. Tata Institute, 1956.

[6] K. Kunen: Set Theory. North-Holland, 1980.

[7] M. Lefranc, Analyse spectrale sur $\mathbb{Z}_{n}$, C. R. Paris 246 (1958), 19511953.

[8] J. F. Ritt: Differential Algebra. The American Mathematical Society Colloquium Publications, Vol. 33, 1950.

[9] J. J. Rotman: An Introduction to the Theory of Groups. Third edition. Wm. C. Brown Publishers, Dubuque, Iowa, 1988.

[10] L. Schwartz, Théorie génerale des fonctions moyenne-périodiques, Ann. of Math. 48 (4) (1947), 857-929.

[11] L. Székelyhidi, The failure of spectral synthesis on some types of discrete Abelian groups, J. Math. Anal. and Applications 291 (2004), 757763.

[12] L. Székelyhidi, Polynomial functions and spectral synthesis, Aequationes Math., 70 (1-2), (2005), 122-130. 
Department of Analysis

Eötvös Loránd University

Budapest, Pázmány Péter sétány 1/C

1117 Hungary

and

Department of Mathematics

University College London

Gower Street, London, WC1E 6BT

England.

e-mail: laczk@cs.elte.hu

Institute of Mathematics

University of Debrecen

Debrecen, P.O. Box 12

4010 Hungary

e-mail: szekely@math.klte.hu 